\title{
Prompt determination of the static stability margins in electrical energy systems equipped with distributed generation plants
}

\author{
$Y u N$ Bulatov $^{1,}$, , $A V$ Kryukov $^{2,3}, K V$ Suslov $^{3}$, and $A V$ Cherepanov $^{2}$ \\ ${ }^{1}$ Bratsk State University, 40, Makarenko st., Bratsk, Russia \\ ${ }^{2}$ Irkutsk State Transport University, 15, Chernyshevsky st., Irkutsk, Russia \\ ${ }^{3}$ Irkutsk National Research Technical University, 83, Lermontova st., Irkutsk, Russia
}

\begin{abstract}
The wide use of distributed generation (DG) technologies in electrical energy systems (EES) requires development of new control algorithms in normal, emergency and postemergency modes. The issues of determining the static aperiodic stability (SAS) margin in EES equipped with DG plants are of particular relevance. These plants can be removed from the consumption centers, which can lead to SAS reserves depletion. The article presents the results of studies aimed at the developing SAS reserves express calculation methods in EES equipped with DG plants. An effective technique to determine stability margins was proposed based on one of the modifications of limiting modes equations. The results of determining SAS for an electrical network with distributed generation plants are presented. Additionally, the simulation of transient processes in the studied EES for various points in the space of the mode's controlled parameters has been carried out in the Matlab system.
\end{abstract}

\section{Introduction}

The issues of determining limit load modes and static aperiodic stability (SAS) margins [13] are relevant when designing and operating electrical energy systems (EES) and have both independent significance and are an integral part of other electrical energy issues related to providing a required level of EES reliability [4].

The issues of determining SAS reserves are of particular relevance in power supply systems equipped with distributed generation (DG) plants, in particular, implemented on the basis of non-traditional renewable energy sources [5-18]. Such plants, as mini hydropower plants and offshore windmill farms can be in a remote location from consumption centers, which leads to SAS reserves depletion [19, 20]. This work considers the technique for situational determining static stability margin in electrical networks with DG plants, in which case limit loads equations are used in the above technique. Situational calculations of stability margins for three-node equivalent circuit with two DG plants and EES connection are provided. Additionally, transient processes simulation is performed.

\footnotetext{
* Corresponding author: bulatovyura@yandex.ru
} 


\section{Problem formulation}

Static stability margin can be determined as Euclidian norm of $\mathbf{K}$ vector [3]:

$$
\mathfrak{I}=\left(\mathbf{K}^{T} \mathbf{K}\right)^{\frac{1}{2}}=\left(\sum_{i=1}^{m} k_{i}^{2}\right)^{\frac{1}{2}}=\left(\sum_{i=1}^{m} \mu_{i}^{2}\left(y_{i L}-y_{i 0}\right)^{2}\right)^{\frac{1}{2}} ; \mu_{i}=\frac{1}{k_{i N} y_{i N O M}},
$$

where $y_{i L}, y_{i 0}$ - values of $i$-th controlled parameter in limit and reference conditions; $k_{i N}$ - norm factor; $y_{\text {iNOM }}$ - parameter rated value $y_{i}$.

In such problem formulation the static stability margin is a distance (in the metric set by the coefficients $\mu_{i}$ ) from the point $\mathbf{Y}_{0}$ to hypersurface $L_{F}$ (Figure 1). Each loading direction $\Delta \mathbf{Y}_{i}$ will correspond to its value $\mathfrak{J}_{i}$ and the search of loading critical direction is required for reliable assessment of stability margin $\mathbf{Y}^{*}(T)=\mathbf{Y}_{0}+T \Delta \mathbf{Y}^{*}$, corresponding to the shortest length $\mathfrak{I}_{\min }$ of $\mathbf{K}$ vector.

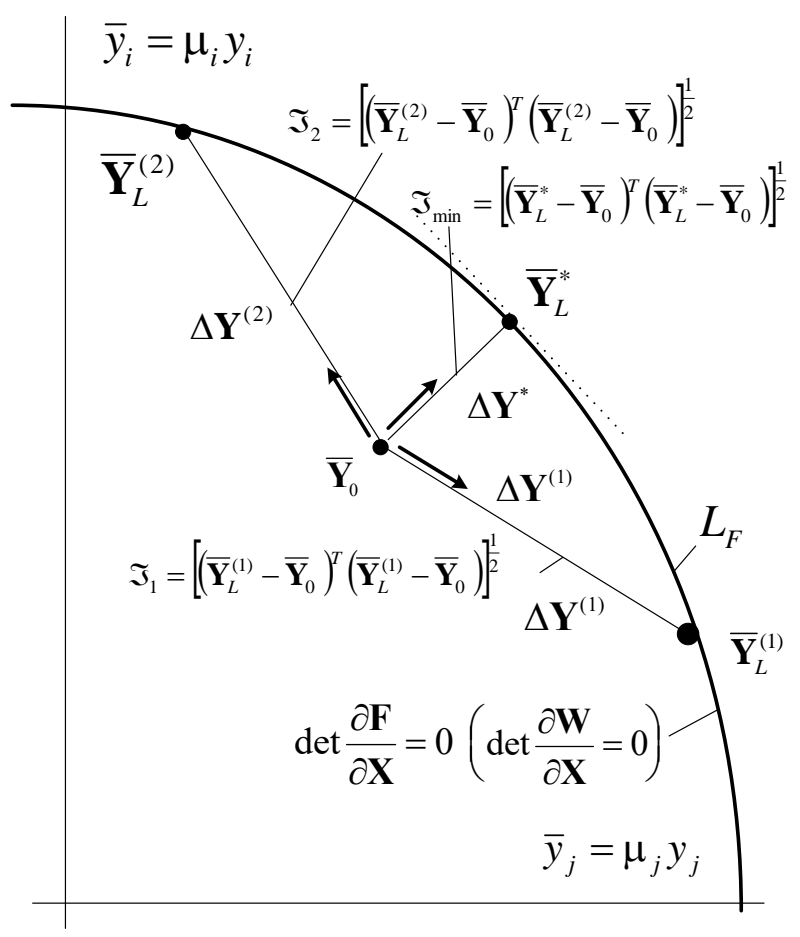

Fig. 1. With respect to stability margin determining.

Based on limit loads equations (LLE) [1,4] technique for determining parameters $\mathbf{Y}_{L}^{*}$, required for reliable assessment of stability margins, can be implemented. 


\section{Technique for operational determining SAS reserves based on limit loads equations}

The problem of assessing the stability margin in the loading critical direction can be formulated as follows:

determine

$$
\mathfrak{I}_{\min }=\min \left(\mathrm{D} \mathbf{Y}^{T} \mathbf{M}^{2} \mathrm{DY}\right)^{\frac{1}{2}}
$$

under constraints

$$
\mathbf{F}\left(\mathbf{X}, \mathbf{Y}_{0}+D \mathbf{Y}\right)=\mathbf{0}
$$

where $\mathbf{F}$ - non-linear vector-function, satisfying steady-state equations (SSE) of EES; $\mathbf{Y}_{0}-$ controlled parameters vector value in the mode, for which the margin is determined; $D \mathbf{Y}=\left[\begin{array}{llll}d y_{1} & d y_{2} & \ldots & d y_{n}\end{array}\right]^{T}$ - vector of incremental variables $\mathbf{Y}_{0}$ which ensure the mode's 'bringing' to hypersurface $L_{F}$, and satisfying the modes limiting with respect to SAS; $\mathbf{M}=\operatorname{diag} \mu_{i}$.

Formally, in addition to condition (2), it would be necessary to introduce the constraint $\operatorname{det} \frac{\partial \mathbf{F}}{\partial \mathbf{X}}=0$. However, as it will be shown below, this constraint has already been set in equations (1) and (2).

To solve the formulated problem (assuming that the limits of stability and the transmitted power coincide), the Lagrange function is written

$$
L\left(\mathbf{X}, \mathbf{Y}_{0}+D \mathbf{Y}, \boldsymbol{\Lambda}\right)=\left(D \mathbf{Y}^{T} \mathbf{M}^{2} D \mathbf{Y}\right)^{\frac{1}{2}}+\mathbf{F}^{T}\left(\mathbf{X}, \mathbf{Y}_{0}+D \mathbf{Y}\right) \boldsymbol{\Lambda}
$$

where $\Lambda$ - the undetermined multiplier vector.

$L$ minimum complies with conditions

$$
\begin{aligned}
& \frac{\partial L}{\partial D \mathbf{Y}}=\mathbf{M}^{2} D \mathbf{Y}\left(D \mathbf{Y}^{T} \mathbf{M}^{2} D \mathbf{Y}\right)^{-\frac{1}{2}}+\left(\frac{\partial \mathbf{F}}{\partial D \mathbf{Y}}\right)^{T} \Lambda=0 \\
& \frac{\partial L}{\partial \mathbf{X}}=\left(\frac{\partial \mathbf{F}}{\partial \mathbf{X}}\right)^{T} \Lambda=0 \\
& \frac{\partial L}{\partial \Lambda}=\mathbf{F}\left(\mathbf{X}, \mathbf{Y}_{0}+D \mathbf{Y}\right)=0
\end{aligned}
$$

This system has two solutions:

1. A trivial one, when it satisfies the initial mode with parameters $\mathbf{X}_{0}, \mathbf{Y}_{0}$, when $\Lambda=[0 \ldots 0]^{T}, D \mathbf{Y}=[0 \ldots 0]^{T}$.

2. Required one, when at least one of the vectors components $\Lambda$ and $D \mathbf{Y}$ is not equal 
to 0 . In this case, the equation $\left(\frac{\partial \mathbf{F}}{\partial \mathbf{X}}\right)^{T} \Lambda=0$ satisfies condition $\operatorname{det}\left(\frac{\partial \mathbf{F}}{\partial \mathbf{X}}\right)^{T}=0$.

Hence, such a solution satisfies the limiting loads hypersurface $L_{F}$. The first equation of the system (3) ensures the shortest distance in metrics set by $\mathbf{M}$ matrix from point $\mathbf{Y}_{0}$ to hypersurface $L_{F}$. The second system equation ensures mode 'output' to hypersurface $L_{F}$ for non-zero $\Lambda$. The third system equation satisfies mode equilibrium.

Geometrically, the system (3) solution is the contact point of hypersurface $L_{F}$ and ellipsoid with center in the point $\mathbf{Y}_{0}$ (Figure 2), described by equation

$$
D \mathbf{Y}^{T} \mathbf{M}^{2} D \mathbf{Y}-\mathfrak{J}_{\min }^{2}=0
$$

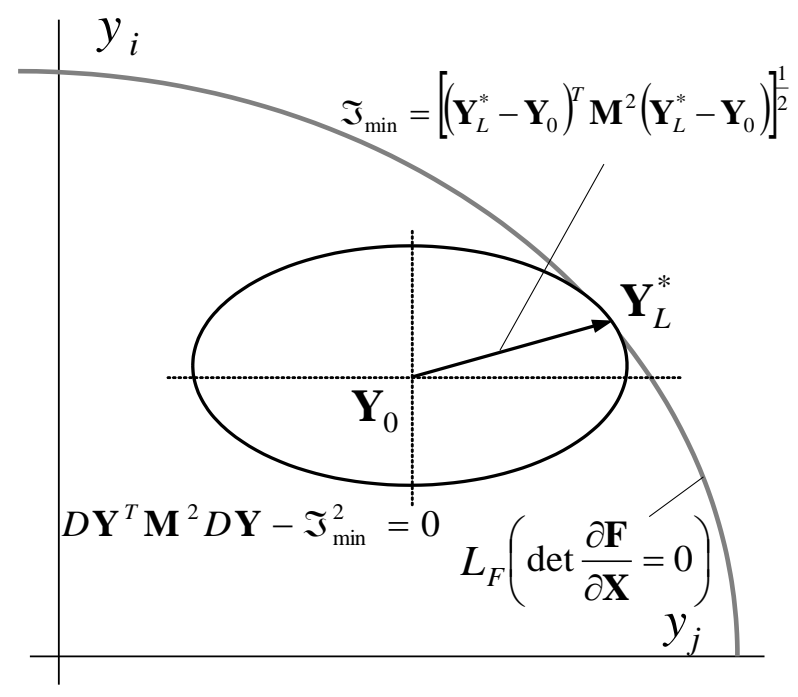

Fig. 2. Geometrical interpretation of weighting critical direction search.

Equations (3) can be represented as

$$
\begin{aligned}
& \frac{\partial L}{\partial D \mathbf{Y}}=\mathbf{M}^{2} D \mathbf{Y}+\left(\frac{\partial \mathbf{F}}{\partial D \mathbf{Y}}\right)^{T} \Lambda\left(D \mathbf{Y}^{T} \mathbf{M}^{2} D \mathbf{Y}\right)^{\frac{1}{2}}=0 \\
& \frac{\partial L}{\partial \mathbf{X}}=\left(\frac{\partial \mathbf{F}}{\partial \mathbf{X}}\right)^{T} \Lambda=0 \\
& \frac{\partial L}{\partial D \mathbf{Y}}=\mathbf{F}\left(\mathbf{X}, \mathbf{Y}_{0}+D \mathbf{Y}\right)=0
\end{aligned}
$$

Since vector $\Lambda$ is determined the accuracy of the multiplier, variables replacement can be made 


$$
\mathbf{R}=\mathfrak{I}_{\min } \boldsymbol{\Lambda}=\left(D \mathbf{Y}^{T} \mathbf{M}^{2} D \mathbf{Y}\right)^{\frac{1}{2}} \boldsymbol{\Lambda}
$$

whereas

$$
\begin{aligned}
& \frac{\partial L}{\partial D \mathbf{Y}}=\mathbf{M}^{2} D \mathbf{Y}+\left(\frac{\partial \mathbf{F}}{\partial D \mathbf{Y}}\right)^{T} \mathbf{R}=0 \\
& \frac{\partial L}{\partial \mathbf{X}}=\left(\frac{\partial \mathbf{F}}{\partial \mathbf{X}}\right)^{T} \mathbf{R}=0 \\
& \frac{\partial L}{\partial D \mathbf{Y}}=\mathbf{F}\left(\mathbf{X}, \mathbf{Y}_{0}+D \mathbf{Y}\right)=0
\end{aligned}
$$

Having determined from the first equation

$$
D \mathbf{Y}=-\mathbf{M}^{-2}\left(\frac{\partial \mathbf{F}}{\partial D \mathbf{Y}}\right)^{T} \mathbf{R}
$$

and, by substituting into the third equation of system (6), one can obtain a system that is LLE modification designed to search for the limiting mode in the critical direction of loading:

$$
\left.\begin{array}{l}
\mathbf{F}\left(\mathbf{X}, \mathbf{Y}_{0}-\mathbf{M}^{-2}\left(\frac{\partial \mathbf{F}}{\partial D \mathbf{Y}}\right)^{T} \mathbf{R}\right)=0, \\
\left(\frac{\partial \mathbf{F}}{\partial \mathbf{X}}\right)^{T} \mathbf{R}=0 .
\end{array}\right\}
$$

If vector components $D \mathbf{Y}$ are introduced to the first group of equations (6) linearly, then $\left(\frac{\partial \mathbf{F}}{\partial D \mathbf{Y}}\right)^{T}=\mathbf{E}$.

This takes place when SSE recorded in Cartesian coordinates system can be represented as:

$$
\begin{aligned}
& f_{2 i-1}(\mathbf{X}, \mathbf{Y})=P_{i 0}+d P_{i}-P_{c i}\left(U_{1}^{\prime}, U_{1}^{\prime \prime}, \ldots U_{p}^{\prime}, U_{p}^{\prime \prime}\right)=0 \\
& f_{2 i}(\mathbf{X}, \mathbf{Y})=Q_{i 0}+d Q_{i}-Q_{c i}\left(U_{1}^{\prime}, U_{1}^{\prime \prime}, \ldots U_{p}^{\prime}, U_{p}^{\prime \prime}\right)=0
\end{aligned}
$$

where $P_{i 0}, Q_{i 0}$ - power injections in the initial mode; $U_{i}^{\prime}, U_{i}^{\prime \prime}$ - real and imaginary components of nodal voltages; $d P_{i}, d Q_{i}$ - vector components $D \mathbf{Y} ; p$ - mains nodes number, apart from balancing. With an implicit dependence of $\mathbf{Y}$ on $\mathbf{X}$, the matrix 
$\left(\frac{\partial \mathbf{F}}{\partial D \mathbf{Y}}\right)^{T}$ is a block-diagonal one.

To solve equations (8), one can use Newton's method; in this case, at each iteration, the following system of linear equations (SLE) is solved:

$$
\left[\begin{array}{ll}
\frac{\partial \mathbf{F}}{\partial \mathbf{X}} & \frac{\partial \mathbf{F}}{\partial \mathbf{R}} \\
\frac{\partial \mathbf{V}}{\partial \mathbf{X}} & \frac{\partial \mathbf{V}}{\partial \mathbf{R}}
\end{array}\right] \cdot\left[\begin{array}{c}
\Delta \mathbf{X} \\
\Delta \mathbf{R}
\end{array}\right]=-\left[\begin{array}{l}
\mathbf{F} \\
\mathbf{V}
\end{array}\right]
$$

where $\frac{\partial \mathbf{F}}{\partial \mathbf{R}}=-\mathbf{M}^{-2}\left(\frac{\partial \mathbf{F}}{\partial D \mathbf{Y}}\right)^{T} ; \frac{\partial \mathbf{V}}{\partial \mathbf{R}}=\left(\frac{\partial \mathbf{F}}{\partial \mathbf{X}}\right)^{T}$.

System (8) can be written as a single vector equation

$$
\mathbf{H}(\mathbf{Z})=\mathbf{0} \text {. }
$$

This equation will satisfy two solutions: $\mathbf{Z}_{0}=\left\{\mathbf{X}_{0}, \mathbf{R}=\mathbf{0}\right\} ; \mathbf{Z}_{L}^{*}=\left\{\mathbf{X}_{L}^{*}, \mathbf{R} \neq \mathbf{0}\right\}$.

Along with nontrivial solutions $\mathbf{Z}_{L}^{*}$, corresponding to the global extremum of the function

$$
\mathfrak{I}_{\text {min }}=\left[\left(\mathbf{Y}_{L}^{*}-\mathbf{Y}_{0}\right)^{T} \mathbf{M}^{2}\left(\mathbf{Y}_{L}^{*}-\mathbf{Y}_{0}\right)\right]^{\frac{1}{2}}
$$

it is possible to reach the points of local extreme of this function.

An effective way to overcome the difficulties associated with the presence of a trivial solution to equations (8) can be implemented by including in these equations a condition requiring that $\mathbf{R}$ vector at the solution locus should not be not zero [4].

This can be done by introducing an indicator variable $\alpha$, by representing system (8) as:

$$
\begin{aligned}
& \mathbf{F}\left[\mathbf{X}, \mathbf{Y}_{0}-\mathbf{M}^{-2}\left(\frac{\partial \mathbf{F}}{\partial D \mathbf{Y}}\right)^{T} \mathbf{R}(1+\alpha)\right]=0 \\
& \mathbf{V}(\mathbf{X}, \mathbf{R})=\left(\frac{\partial \mathbf{F}}{\partial \mathbf{X}}\right)^{T} \mathbf{R}=0 \\
& U(\mathbf{R})=\mathbf{R}^{T}\left(\frac{\partial \mathbf{F}}{\partial D \mathbf{Y}}\right) \mathbf{M}^{-2}\left(\frac{\partial \mathbf{F}}{\partial D \mathbf{Y}}\right)^{T} \mathbf{R}-\mathfrak{I}_{1}{ }^{2}=0
\end{aligned}
$$

where $\mathfrak{J}_{1} \neq 0$ - is the fictitious reserve value.

In this case, the SLE solved at each iteration will have the form: 


$$
\left[\begin{array}{ccc}
\frac{\partial \mathbf{F}}{\partial \mathbf{X}} & \frac{\partial \mathbf{F}}{\partial \mathbf{R}} & \frac{\partial \mathbf{F}}{\partial \alpha} \\
\frac{\partial \mathbf{V}}{\partial \mathbf{X}} & \frac{\partial \mathbf{V}}{\partial \mathbf{R}} & 0 \\
0 & \frac{\partial U}{\partial \mathbf{R}} & 0
\end{array}\right] \cdot\left[\begin{array}{c}
\Delta \mathbf{X} \\
\Delta \mathbf{R} \\
\Delta \alpha
\end{array}\right]=-\left[\begin{array}{c}
\mathbf{F} \\
\mathbf{V} \\
U
\end{array}\right],
$$

where $\frac{\partial \mathbf{F}}{\partial \alpha}=-\mathbf{M}^{-2}\left(\frac{\partial \mathbf{F}}{\partial D \mathbf{Y}}\right)^{T} \mathbf{R}$.

In an abridged form, the last system can be written as follows $\frac{\partial \mathbf{H}}{\partial \mathbf{Z}} \Delta \mathbf{Z}=-\mathbf{H}$.

The value of the stability margin $\mathfrak{I}$ is determined after the end of the iteration process using the formula $\mathfrak{I}=(1+\alpha) \sqrt{\mathbf{R}^{T}\left(\frac{\partial \mathbf{F}}{\partial D \mathbf{Y}}\right) \mathbf{M}^{-2}\left(\frac{\partial \mathbf{F}}{\partial D \mathbf{Y}}\right)^{T} \mathbf{R}}$.

Determination of the SAS reserves based on the above described approach was carried out in relation to the network $[19,20]$ diagram, the general layout and diagram of which are shown in Figure 3. The DG plants rated powers are assumed equal to 24 MW. Simulation results are provided in Figure 4 and in Table 1.

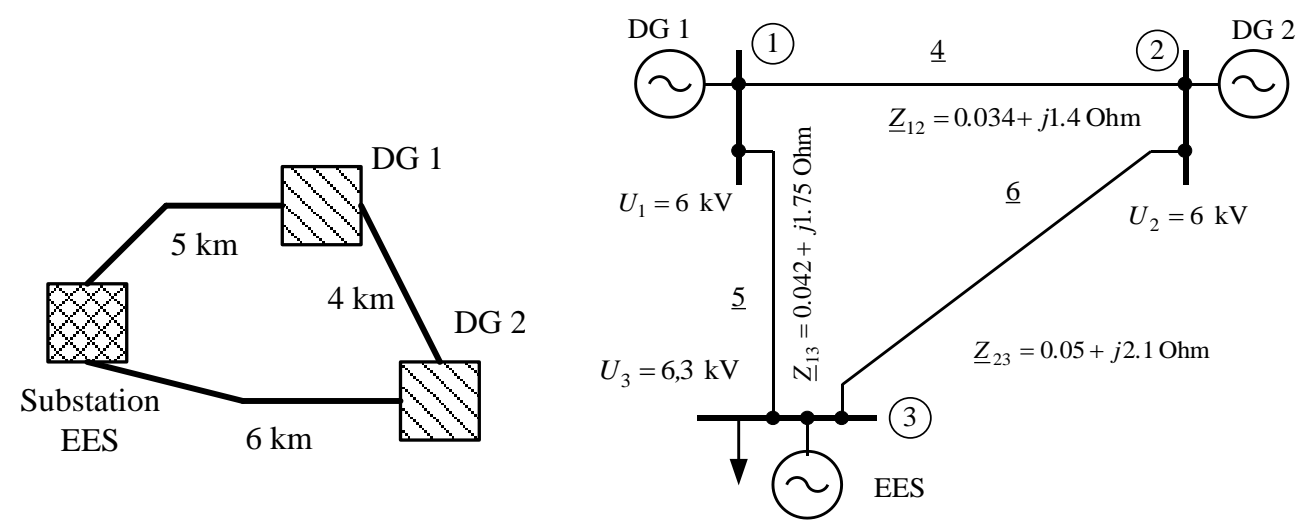

(a)

(b)

Fig. 3. Network diagram. 


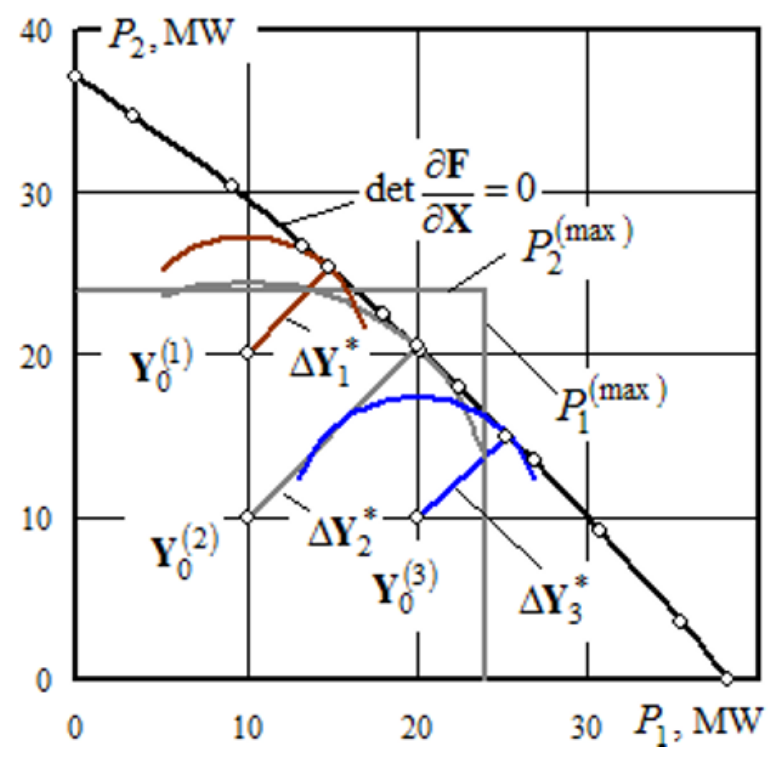

Fig. 4. Results of determining the limiting modes in the critical direction of loading: circles arcs correspond to the equations $D \mathbf{Y}^{T} \mathbf{M}^{2} D \mathbf{Y}-\mathfrak{I}_{\min }^{2}=0$ when $\mathbf{M = E}$.

Table 1. Stability margins.

\begin{tabular}{|c|c|c|c|c|}
\hline № & $\boldsymbol{P}_{\mathbf{1 0}}, \mathbf{M W}$ & $\boldsymbol{P}_{\mathbf{2 0}}, \mathbf{M W}$ & $\mathfrak{I}, \mathbf{M W}$ & $\mathfrak{I}^{*}, \boldsymbol{\%}$ \\
\hline 1 & 10 & 20 & 7.2 & 55 \\
\hline 2 & 20 & 10 & 7.38 & 58 \\
\hline 3 & 10 & 10 & 14.5 & 145 \\
\hline 4 & 20 & 20 & 0.34 & 1.7 \\
\hline
\end{tabular}

Note:

$$
\mathfrak{I}=\sqrt{\sum_{\mathrm{k}=1}^{2}\left(P_{L 1}-P_{01}\right)^{2}}=(1+\alpha) \sqrt{\mathbf{R}^{T}\left(\frac{\partial \mathbf{F}}{\partial D \mathbf{Y}}\right) \mathbf{M}^{-2}\left(\frac{\partial \mathbf{F}}{\partial D \mathbf{Y}}\right)^{T} \mathbf{R}} ;
$$

$\mathfrak{J}^{*}=100 \sqrt{\sum_{\mathrm{k}=1}^{2}\left(\frac{P_{L 1}-P_{01}}{P_{01}}\right)^{2}}$.

The results obtained allow us to conclude that an efficient technique for analyzing static aperiodic stability in an EES equipped with DG plants can be implemented on the LLE basis, which is applicable in design problems, as well as operational and emergency control (OC and EC). The nondegeneracy of the LLE Jacobi matrix at the solution locus [4] ensures reliability of results obtaining, which is very important for OC and EC problems solution.

\section{Determining stability margins based on limiting modes equations with increased non-linearity}


Parameters of the limiting mode in critical (the most dangerous) loading direction $\Delta \mathbf{Y}_{c r}$ can be found from solution of the following equations system [4]:

$$
\mathbf{V}(\mathbf{X})=\left(\frac{\partial \mathbf{F}}{\partial \mathbf{X}}\right)^{T} \mathbf{F}\left(\mathbf{X}, \mathbf{Y}_{0}\right)=0
$$

However, when using these equations, significant computational difficulties arise due to the presence of a trivial solution $\mathbf{X}=\mathbf{X}_{0}$, corresponding to the initial mode. To overcome this difficulty, an additional variable and the equation can be introduced into system (15)

$$
\left.\begin{array}{l}
\mathbf{V}(\mathbf{X})=\left(\frac{\partial \mathbf{F}}{\partial \mathbf{X}}\right)^{T} \mathbf{F}\left(\mathbf{X}, \mathbf{Y}_{0}\right)=0 \\
u(\mathbf{X}, c)=\mathbf{F}^{T}\left(\mathbf{X}, \mathbf{Y}_{0}\right) \cdot \mathbf{F}\left(\mathbf{X}, \mathbf{Y}_{0}\right)-1-c^{2}=0
\end{array}\right\}
$$

where $c$ is a variable that determines the stability margin value.

Simulation results are provided in Figure 5 and in Table 2.

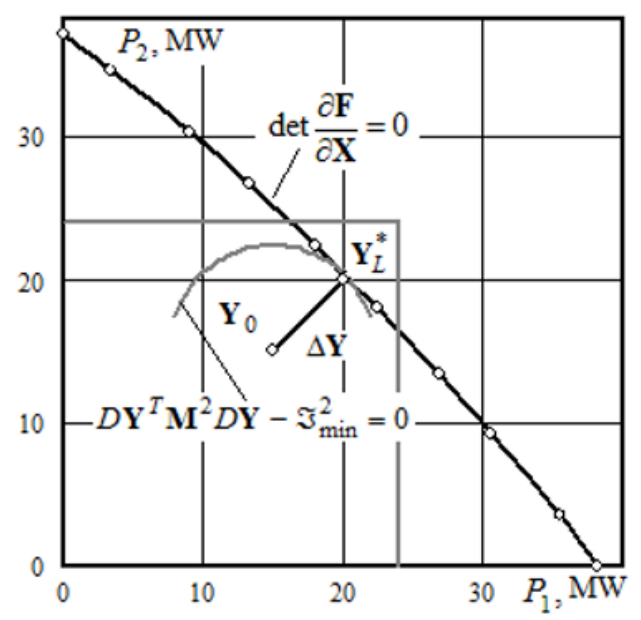

Fig. 5. Results of determining the limiting modes in the critical direction of loading.

Table 2. Stability margins.

\begin{tabular}{|c|c|c|c|c|}
\hline № & $\boldsymbol{P}_{\mathbf{1 0}}, \mathbf{M W}$ & $\boldsymbol{P}_{\mathbf{2 0}}, \mathbf{M W}$ & $\mathfrak{I}, \mathbf{M W}$ & $\mathfrak{I}^{*}, \boldsymbol{\%}$ \\
\hline 1 & 5 & 5 & 21.52 & 430 \\
\hline 2 & 10 & 20 & 7.2 & 55 \\
\hline 3 & 10 & 25 & 3.47 & 25 \\
\hline 4 & 15 & 15 & 7.4 & 49 \\
\hline 5 & 19 & 19 & 1.8 & 9 \\
\hline 6 & 25 & 10 & 3.74 & 28 \\
\hline
\end{tabular}


Note: $\mathfrak{I}=\sqrt{\sum_{\mathrm{k}=1}^{2}\left(P_{L 1}-P_{01}\right)^{2}} ; \mathfrak{J}^{*}=100 \sqrt{\sum_{\mathrm{k}=1}^{2}\left(\frac{P_{L 1}-P_{01}}{P_{01}}\right)^{2}}$.

Thus, based on equations (16), an alternative approach to solving the problem of SAS reserves operational determining can be implemented.

\section{Transient processes simulation results}

Two mini-hydroelectric power plants with a rated power of $24 \mathrm{MW}$ each, operating from the synchronous generators, are used as DG plants in the system under study. The operating modes of the network under study when an additional load is connected at the EES substation, as well as in emergency mode, for which lines 1-3 are exemplified, imply the implementation of power delivery from each generator to the receiving system (node 3 in Figure 3). The diagram of the network model under study, developed using the Simulink and SimPowerSystems simulation packages, is provided in Figure 6. The model used the standard units of the specified Matlab system modeling packages. In this case, thyristor excitation systems of synchronous generators (Excitation System1 and Excitation System2 units) were simulated by the first-order aperiodic link with transfer function $\frac{1}{0.025 s+1}$. The Hydraulic Turbine unit in Figure 6 consists of the main servomotor with the proportionalplus-integral controller, speed limiter and a hydraulic turbine itself. The mathematical model of the main servomotor with the controller is represented by the following transfer function:

$$
W(s)=\frac{T_{i} s+1}{\left(T_{i} s+1\right) \cdot\left(T_{s} s+1\right)+n_{i} \cdot T_{i} s},
$$

where $n_{i}$ - proportional-plus-integral controller amplification factor (was assumed equal to 10 r.u. (relative units) when simulating); $T_{i}$ - the control unit time constant (was assumed equal to $0.1 \mathrm{~s}$ ); $T_{s}$ - servo motor time constant (was assumed equal to $0.25 \mathrm{~s}$ ).

Hydraulic turbine was modeled by the activation function providing for hydraulic shock possibility [21]:

$$
W_{G}(s)=\frac{1-a \cdot T_{G} s}{1+0.5 a \cdot T_{G} s}
$$

where $T_{G}$-hydroturbine time constant (was assumed equal to $0.344 \mathrm{~s}$ when modeling); $a$ - position of hydroturbine wicket gate opening (assumes values within the range $0 \ldots 1$ ). 


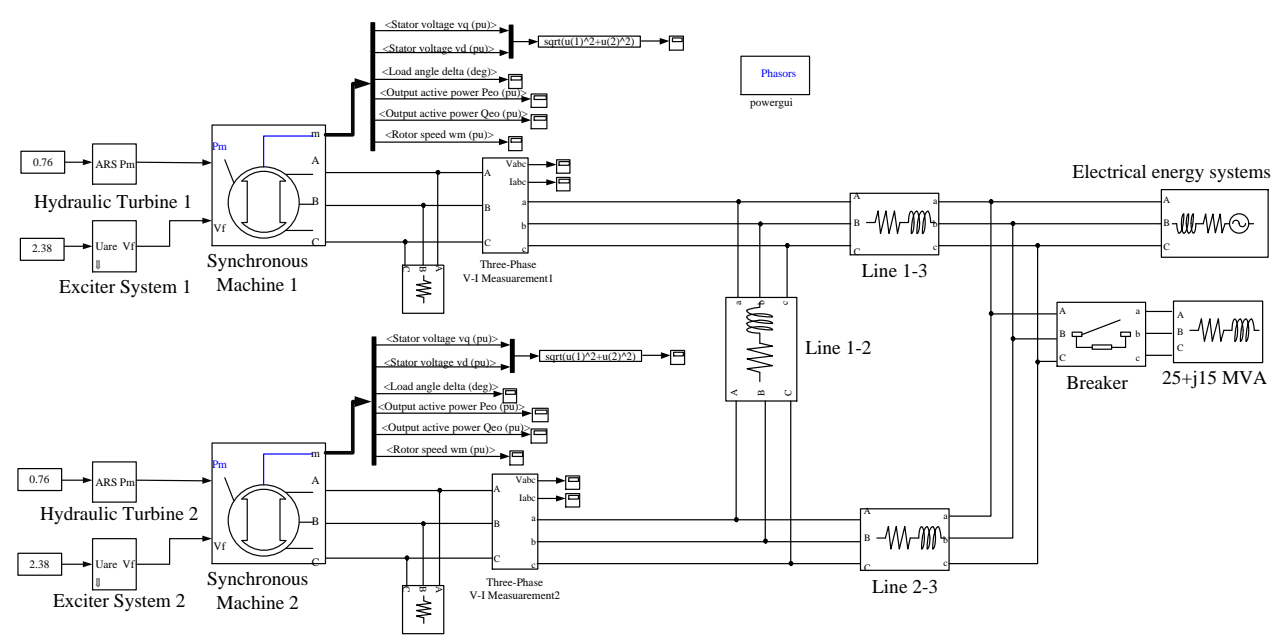

Fig. 6. The model diagram of network under study in Matlab.

The DG plants synchronous generators were simulated by the standard unit of the SymPowerSystems package of the MATLAB - Synchronous Machine pu Fundamental system, with the unit having the following parameters: rated power - 24 MVA; electric line voltage $6 \mathrm{kV}$; generator voltage and current frequency - $50 \mathrm{~Hz}$; stator resistance per phase $R_{s}=0.008979$ r.u.; stator leakage inductance $L_{l}=0.05$ r.u.; direct-axis magnetizing inductance viewed from stator $L_{m d}=2.35$ r.u.; quadrature-axis magnetizing inductance viewed from stator $L_{m q}=1.72$ r.u.; field resistance $R_{f}=0.0021$ r.u.; field leakage inductance $L_{l f d}=0.511$ r.u.; inertia coefficient $\mathrm{H}=2.8485 \mathrm{~s}$; friction factor $\mathrm{F}=0.0092$ r.u.; number of pole pairs $\mathrm{p}=3$.

Power lines (Line units in Figure 6) are simulated by a series-connected RL circuit, the resistances of which are shown in Figure 3.

Transient processes simulation was carried out with a short-term switching on of a load of $25+\mathrm{j} 15 \mathrm{MV} \cdot \mathrm{A}$ at the EES substation. For the purity of the experiment, the generators of the DG plants were simulated without automatic voltage regulators (AVR) and automatic speed regulators (ASR). The simulation results in the form of powers, rotor speeds and DG1 plant generator voltages oscillograms are shown in Figure 7. Oscillograms of DG2 generator parameters are similar.

The computer modeling results confirm the correctness of EES stability margins determining with DG plants. For parameters values of the mode located on the boundary of the SAS region, and the disturbance introduced into the EES via connecting an additional load, the expected loss of the DG plants generators stability is observed (Figure 7, b). 

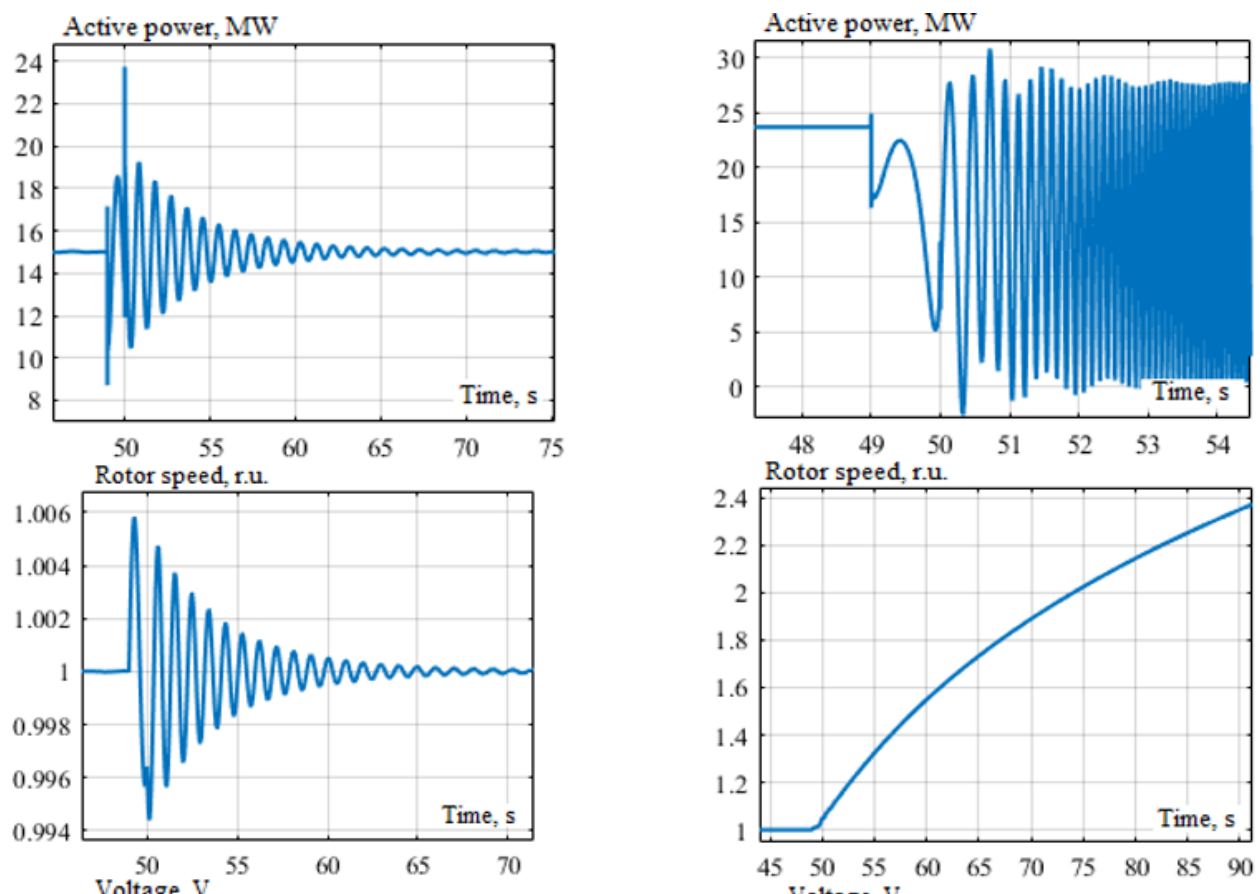

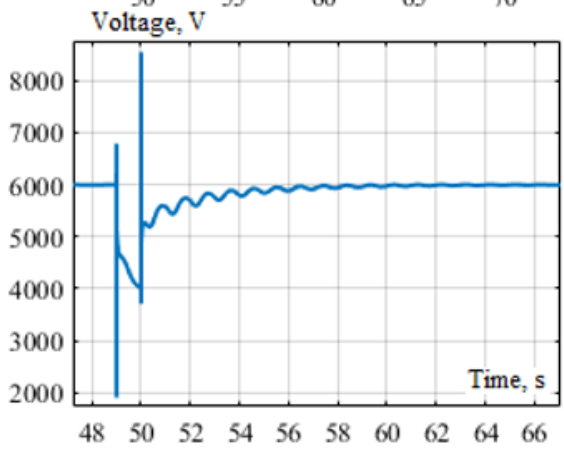

(a)

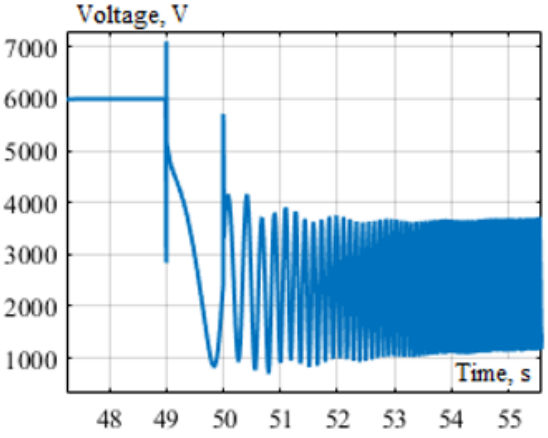

(b)

Fig. 7. Oscillograms of power, rotor speed and voltage of the DG1 plant generator at a short-term connecting of a powerful load in the complete diagram of the network under study: (a) $P_{1}=15 \mathrm{MW}$, $P_{2}=15 \mathrm{MW}$ (stable mode); (b) $P_{1}=23.7 \mathrm{MW}, P_{2}=17 \mathrm{MW}$ (unstable mode).

Thus, the dynamic modeling of the electrical network under study with DG plants confirms the stability regions which are calculated using the limit modes equations.

\section{Conclusions}

The following conclusions can be formulated based on the performed theoretical analysis and computer-aided modeling:

1. An effective technique is proposed for the operational determining of stability margins regions in an EES equipped with DG plants. The nondegeneracy of the LLE Jacobi matrix 
of limit loads equations at the solution loci ensures the reliability of results obtaining, which is very important in solving the issues of operational and, especially, emergency control.

2. The results of determining SAS reserves for an electrical network with distributed generation plants are presented. The computer modeling results confirm the correctness of EES stability margins calculation using the proposed technique.

3. An alternative approach to solving the issue of SAS reserves determining can be implemented based on the limiting modes equations of with increased non-linearity.

\section{Acknowledgements}

The research was carried out within the state assignment of the Ministry of Science and Higher Education of Russian Federation on the topic "Improving the quality of electrical energy and electromagnetic safety in power supply systems of railways equipped with Smart Grid devices by using methods and means of mathematical modeling based on phase coordinates."

\section{References}

1. Kontorovich A M, Kryukov A V 1987 Proceedings of the Academy of Sciences of the USSR. Power Engineering 325

2. Ayuev B I, Davydov V V, Erokhin P M 2016 IEEE Trans. Power Syst. 314525 doi.org/10.1109/TPWRS.2016.2538299

3. Makarov Yu V, Ma J, Dong Zh Y 2007 Proceedings of the IEEE Power Engineering Society General Meeting 1 doi.org/10.1109/PES.2007.385897

4. Kryukov A V 2012 Limiting regimes of electric power systems (Irkutsk) p 236

5. Rugthaicharoencheep N, Auchariyamet S 2012 International Journal of Electrical, Computer, Energetic, Electronic and Communication Engineering 6385 doi.org/10.5281/zenodo. 1327636

6. Buchholz B M, Styczynski Z 2014 Smart Grids - Fundamentals and Technologies in Electricity Networks (Heidelberg New York Dordrecht, London: Springer) p 396

7. Magdi S M, Fouad M AL-Sunni 2015 Control and Optimization of Distributed Generation Systems (Cham: Springer International Publishing, Imprint: Springer) $\mathrm{p}$ 578

8. Barker Ph P, Mello R W 2000 IEEE PES Summer Meeting, Seattle, WA, USA 222 doi.org/10.1109/PESS.2000.868775

9. Voropai N I, Stychinsky Z A 2010 Renewable energy sources: theoretical foundations, technologies, technical characteristics, economics (Magdeburg: Otto-von-GuerickeUniversität) p 223

10. Saleh M S, Althaibani A, Esa Y, Mhandi Y, Mohamed A A 2015 Proceedings on International Conference on Smart Grid and Clean Energy Technologies (ICSGCE) 195

11. Mohsen F N, Amin M S, Hashim H 2013 IEEE 7th International Power Engineering and Optimization Conference (PEOCO). doi.org/10.1109/PEOCO.2013.6564586

12. Wang J, Huang A Q, Sung W, Liu Y, Baliga B J 2009 IEEE Industrial Electronics Magazine 3 16 doi.org/10.1109/MIE.2009.932583 
13. Shen X, Zhu S, Zheng J, Han Y, Li Q, Nong J, Shahidehpouret M 2015 Power \& Energy Society General Meeting. IEEE Conference Publications doi.org/10.1109/PESGM.2015.7286069

14. Martínez Ceseña E A, Capuder T, Mancarella P 2016 IEEE Transaction on Smart Grid 7348 doi.org/10.1109/PESGM.2016.7741088

15. Huang A Q, Crow M L, Heydt G T, Zheng J P 2010 S. J. N. I. S. Proceedings of the IEEE doi.org/10.1109/JPROC.2010.2081330

16. Olivares D, et al 2014 IEEE Transactions on Smart Grid 51905

17. Ackermann T, Anderson G, Söder L 2001 Electric Power Systems Research 57195 doi.org/10.1016/S0378-7796(01)00101-8

18. Ellabban O, Abu-Rub H, Blaabjerg F 2014 Renewable and Sustainable Energy Reviews 39748 doi.org/10.1016/J.RSER.2014.07.113

19. Bulatov Yu N, Kryukov A V 2019 Energy Systems Research 268

20. Bulatov Yu, Kryukov A, Suslov K, Shamarova N 2019 Proceedings of the 10th International Scientific Symposium on Electrical Power Engineering, ELEKTROENERGETIKA 38

21. Anderson P M, Fouad A A 2003 Power System Control and Stability (IEEE Press) p 688 